SLAC-PUB-14028

\title{
Misaligned Discs as Obscurers in Active Galaxies
}

Astrophysical Journal, 2010

\author{
Andy Lawrence ${ }^{1}$ \\ Institute for Astronomy, University of Edinburgh, \\ Royal Observatory, Blackford Hill, Edinburgh EH9 3HJ, U.K. \\ and \\ Martin Elvis \\ Harvard-Smithsonian Center for Astrophysics, \\ 60 Garden Street, Cambridge, MA 02138
}

\begin{abstract}
We review critically the evidence concerning the fraction of Active Galactic Nuclei (AGN) which appear as Type 2 AGN, carefully distinguishing strict Type 2 AGN from both more lightly reddened Type 1 AGN, and from low excitation narrow line AGN, which may represent a different mode of activity. Low excitation AGN occur predominantly at low luminosities; after removing these, true Type 2 AGN represent $58 \pm 5 \%$ of all AGN, and lightly reddened Type 1 AGN a further $\sim 15 \%$. Radio, IR, and volume-limited samples all agree in showing no change of Type 2 fraction with luminosity. X-ray samples do show a change with luminosity; we discuss possible reasons for this discrepancy. We test a very simple picture which produces this Type 2 fraction with minimal assumptions. In this picture, infall from large scales occurs in random directions, but must eventually align with the inner accretion flow, producing a severely warped disk on parsec scales. If the re-alignment is dominated by tilt, with minimal twist, a wide range of covering factors is predicted in individual objects, but with an expected mean fraction of Type 2 AGN of exactly $50 \%$. This "tilted disc" picture predicts reasonable alignment of observed nuclear structures on average, but with distinct misalignments in individual cases. Initial case studies of the few well resolved objects show that such misalignments are indeed present.
\end{abstract}

Subject headings: galaxies:active - galaxies:nuclei - quasars: general - accretion, accretion disks

\section{Introduction}

The standard Unified Scheme for Active Galactic Nuclei (AGN) includes a central continuum source; a region somewhat further out emitting broad emission lines ; a dusty rotating "torus" on parsec scales; and gas emitting narrow emission lines on a scale of tens to hundreds of parsecs, ionised through the open cone defined by the torus edge (Lawrence \& Elvis 1982; Antonucci \& Miller 1985; Krolik \& Begelman 1986, 1988; Urry \& Padovani

\footnotetext{
${ }^{1}$ Visiting Scientist, Kavli Institute for Particle Astrophysics and Cosmology (KIPAC), Stanford University
}

1995). In this picture, Type 2 AGN are those seen sideways through the obscuring torus, so that one sees only the narrow lines and the IR emission produced by reprocessing of the continuum source by the torus. The torus needs to be geometrically thick $(H / R \sim 1)$. The shape and amplitude of the X-ray background (e.g. Gilli et al. (2007)), and the observed narrowness of emission line cones, appear to require that obscured (Type 2) AGN substantially outnumber unobscured (Type 1) AGN, by a factor $\sim 4$, so that the torus must subtend an angle of $65^{\circ}$ seen from the central source (e.g. Risaliti et al. (1999)).

There is little doubt about the essence of the 
Unified Scheme concept - that most Type 2 AGN are the result of obscuration of the inner nucleus by some kind of flattened geometrically thick structure (Lawrence \& Elvis 1982). It is less clear that the specific rotating molecular torus model is correct. As recognised by Krolik \& Begelman (1988), it is extremely difficult to maintain a cold rotating structure in a geometrically thick state, even if made of discrete clouds, or puffed up by radiation pressure (Krolik 2007). Three alternative ways have been suggested to form geometrically thick obscuring structures. (i) A starburst disk in the host galaxy, kept turbulent and thick by supernova heating (Fabian et al. 1998; Wada \& Norman 2002; Thompson et al. 2005; Ballantvne 2008). (ii) Dust bearing outflowing winds (Elvis et al. 2002; Elitzur \& Shlosman 2006); see also Fig. 8 of Risaliti et al. (2002). (iii) Warped disks (Phinnev 1989; Sanders et al. 1989; Greenhill et al. 2003; Nayakshin 2005; Lawrence 2007).

In this paper we examine some specific predictions of the warped disk idea, common to all such models, regardless of their physical cause, under the simplifying assumption of misaligned fuelling from completely random directions (Volonteri et al. 2007). We begin by looking critically at the issue of how many obscured AGN there really are (section 2). Next we review what we know about parsec scale structures and gas flow in AGN (section 3). We then test the predictions of the simplest misaligned disc models (section 4), and finally discuss the general implications of a misaligned disc interpretation (section 5).

\section{The Type 2 fraction, $f_{2}$}

Many authors use the terminology "Type 2 AGN" to mean any AGN showing signs of obscuration. In this paper we use the standard optical definition of a Type 2 AGN - that no broad emission lines or strong blue continuum are seen, while the narrow ( $300-1000 \mathrm{~km} \mathrm{~s}^{-1}$ FWHM) lines are prominent. However, where possible we also track the numbers of lightly reddened broad-line AGN, which show weak broad $\mathrm{H} \alpha$ (see discussion below). These can then be grouped with Type 2 or Type 1 AGN according to what hypothesis one wishes to test. Assessing what is and is not a Type 2 AGN can be difficult, for several reasons. (i) Low quality spectra can result in weak broad lines being missed.

(ii) Work reporting $X$-ray surveys often takes the existence of X-ray absorption as the definition of an "obscured" AGN, but in fact X-ray absorption can occur in the absence of optical extinction. Conversely, objects with very heavy optical extinction, such that the corresponding X-ray column is $\mathrm{N}_{H}>10^{24} \mathrm{~cm}^{-2}$ (so called "Compton Thick" objects) may not be visible in X-ray surveys at all. The generic term "obscuration" can hide some important distinctions; optical and Xray classifications seem to disagree in around $20 \%$ of cases (Tozzi et al. 2006).

(iii) Modest reddening (i.e. $\left.\mathrm{A}_{V} \sim 1-3\right)$ can remove broad lines in the blue, resulting in what is often referred to in the literature as objects of Type 1.8 or 1.9 (Osterbrock \& Koski 1976). These are "obscured" AGN, but they are not Type 2 AGN, and typically have X-ray columns $\mathrm{N}_{H} \sim 10^{22}$ $\mathrm{cm}^{-2}$, a hundred times smaller than true Type 2 AGN. To keep this distinction as clear as possible we refer to them below as "reddened Type $1 \mathrm{~s}$ " or Type 1R, and track their numbers separately where these are reported. (The same terminology has been used recently by Lacy et al. (2007).) The separation between Type $1 \mathrm{R}$ and Type 2 corresponds to approximately $\mathrm{A}_{V} \sim 5$, beyond which broad lines are extremely hard to detect in the optical; however this classification is especially sensitive to data quality, and to redshift. There may simply be a distribution of extinction values, but it has been argued that there are two physically distinct "heavy" and "light" obscuring regions, with the latter related to the host galaxy, and the former to the postulated nuclear "torus" (see Maiolino \& Rieke (1995) and discussion below).

(iv) The nature of LINERs which appear in many samples is still unclear. Hopkins et al. (2009) have recently made the case that at low luminosities in particular, the number of obscured AGN may have been substantially over-estimated, both because of data quality, and because of the prevalence of LINERs.

Given these problems, it is particularly important to concentrate on statistics from reliable samples, and those that have minimum possible or well understood extinction bias. Table 1 summarises statistics from the various samples discussed be- 
low. Where authors report the number of Type 1R objects, we tabulate this; otherwise they are assumed to be included in the quoted number of Type 1 objects. However, some objects classified as Type 2 may eventually turn out to be Type $1 R$. We also tabulate, where known, the number of low-excitation AGN as distinct from classical highexcitation Type 2 AGN. (Table 1 refers to these as "Type L"). This fraction has an even higher degree of uncertainty.

\subsection{Radio selected AGN}

In principle, selection of AGN by low-frequency radio emission is a safe method, being independent of both nuclear obscuration and beaming. (Of course this method only selects a subset of all AGN, those that are radio-loud.) Lawrence (1991) showed that $f_{2}$ apparently changes systematically with radio luminosity in the $3 \mathrm{CR}$ sample, from $f_{2} \sim 0.5$ at $\mathrm{L}_{178}=10^{29} \mathrm{~W} \mathrm{~Hz}^{-1}$, to $f_{2}>0.9$ at $\mathrm{L}_{178}=10^{25} \mathrm{~W} \mathrm{~Hz}{ }^{-1}$. However, several authors, while confirming the luminosity effect in other low-frequency radio samples, have pointed out that most of the non-quasar low-luminosity radio galaxies do not look like classical Type 2 AGN at all; they have low-excitation, and usually very weak, emission lines (Laing et al. 1994; Willott et al. 2000; Grimes et al. 2004). A number of authors, using $3 \mathrm{C}, 6 \mathrm{C}$, and $7 \mathrm{C}$ samples, have argued that such objects are not obscured Type 1 AGN at all, and represent a different mode of nuclear activity. They have optical cores dominated by synchrotron emission, no sign of a midIR excess, and negligible nuclear X-ray emission, suggesting that there is no torus-like structure to extinguish the optical core, to reprocess hidden power, or to scatter hidden power into the line of sight (Chiaberge et al. 1999; Willott et al. 2000; Whysong \& Antonucci 2004; Ogle et al. 2006; Hardcastle et al. 2006, 2009; Dicken et al. 2009).

In Table 1, we report statistics from the studies of Willott et al. (2000) (ref 1) and Ogle et al. (2006) (ref 2), as these authors explicitly correct for likely Type L objects. They are consistent in showing $f_{2} \sim 0.6$. The Type L objects are nearly all at low radio power; Willott et al. (2000) report that after removing them there is no evidence for a luminosity dependence of $\mathrm{f}_{2}$.

However, these studies did not explicitly report numbers of Type $1 \mathrm{R}$ objects. We know that this is significant issue; a careful study of 8 Narrow Line Radio Galaxies at Paschen $\alpha$ by Hill et al. (1996) converted 3 of these to reddened Broad Line Radio Galaxies, and several other such objects are known (Carleton et al. 1984; Goodrich \& Cohen 1992; Economou et al. 1995). Most objects in faint radio samples are at moderately high redshift so the available spectra are in the rest-frame blue; it is likely therefore that the value of $f_{2} \sim 0.6$ is a significant overestimate.

\subsection{Mid-infrared selected AGN}

A second reliable selection method is the midIR $(5-20 \mu \mathrm{m})$, where the obscuring material reradiates its absorbed energy, where stellar contamination is minimal, and where emission by the cooler dust surrounding star forming regions is also relatively weak. Mid-IR emission should be close to being orientation independent, although there are some signs that the emitting region may be slightly optically thick at $12 \mu \mathrm{m}$ (Buchanan et al. 2006). Table 1 shows numbers from three samples - a spectroscopic study of Spitzer $24 \mu \mathrm{m}$ sources (Lacy et al. (2007), ref 3 ); the revised IRAS $12 \mu \mathrm{m}$ sample (Rush et al. (1993), ref 4) ; and the $60 \mu \mathrm{m}$ IRAS "warm" sample (de Grijp et al. (1992), ref 5). Overall, they show $f_{2} \sim 0.5-0.6$. The Rush et al. (1993) sample classifies many objects as LINERs, which as with the radio samples above, dominate at low luminosities. Removing these, there is no sign of a luminosity dependence of $\mathrm{f}_{2}$ (see discussion in section 2.6). Lacy et al. (2007) provide the number of Type $1 \mathrm{R}$ objects, which is $16 \%$ of their sample.

\subsection{Nearby galaxy spectroscopic samples}

A third reliable method is from complete spectroscopic surveys of nearby galaxies, which are volume limited down to quite small galaxy luminosities. In Table 1 we show results from two studies; that of Maiolino \& Rieke (1995) (ref 6), who examined spectral types for AGN for a sample constructed from the Revised Shapley Ames (RSA) catalogue; and the comprehensive Palomar spectroscopic study of nearby galaxies by Ho et al. (1997) (HFS, ref 7). Maiolino \& Rieke (1995) exclude LINERs, but do provide a Type 1R number, which is large, at $20 \%$ of the sample. This sample provides an interesting lesson in how one 
TABLE 1

AGN Type statistics

\begin{tabular}{|c|c|c|c|c|c|c|c|}
\hline Sample & Waveband & Ref & Total & $\begin{array}{c}\text { Type-L } \mathrm{L}^{\mathrm{a}} \\
\mathrm{f}_{L}\end{array}$ & Type- $1^{\mathrm{b}}$ & $\begin{array}{c}\text { Type-1R } \\
\mathrm{f}_{1 R}\end{array}$ & $\begin{array}{c}\text { Type-2 } \\
\mathrm{f}_{2}\end{array}$ \\
\hline $3 \mathrm{C} / 6 \mathrm{C} / 7 \mathrm{C}$ & radio & $(1)$ & 323 & $\begin{array}{c}107 \\
0.33 \pm 0.04\end{array}$ & 86 & & $\begin{array}{c}130 \\
0.6 \pm 0.07\end{array}$ \\
\hline $3 \mathrm{CRR}$ & radio/Mid-IR & $(2)$ & 42 & $\begin{array}{c}17 \\
0.4 \pm 0.12\end{array}$ & 11 & & $\begin{array}{c}14 \\
0.56 \pm 0.19\end{array}$ \\
\hline Spitzer & Mid-IR & $(3)$ & 77 & $\begin{array}{c}7 \\
0.09 \pm 0.03\end{array}$ & 36 & $\begin{array}{c}11 \\
0.16 \pm 0.05\end{array}$ & $\begin{array}{c}34 \\
0.49 \pm 0.1\end{array}$ \\
\hline IRAS- $12 \mu \mathrm{m}$ & Mid-IR & $(4)$ & 145 & $\begin{array}{c}29 \\
0.2 \pm 0.04\end{array}$ & 53 & & $\begin{array}{c}63 \\
0.54 \pm 0.08\end{array}$ \\
\hline IRAS-warm & Mid/Far-IR & $(5)$ & 226 & $\begin{array}{c}5 \\
0.02 \pm 0.01\end{array}$ & 80 & & $\begin{array}{c}141 \\
0.64 \pm 0.07\end{array}$ \\
\hline RSA & optical & $(6)$ & 84 & & 37 & $\begin{array}{c}17 \\
0.2 \pm 0.05\end{array}$ & $\begin{array}{c}47 \\
0.56 \pm 0.1\end{array}$ \\
\hline Palomar-V1 ${ }^{\mathrm{c}}$ & optical & $(7)$ & 218 & $\begin{array}{c}167 \\
0.77 \pm 0.08\end{array}$ & 21 & & $\begin{array}{c}30 \\
0.59 \pm 0.14\end{array}$ \\
\hline Palomar-V2d & & & 218 & $\begin{array}{c}122 \\
0.56 \pm 0.06\end{array}$ & 66 & & $\begin{array}{c}30 \\
0.31 \pm 0.07\end{array}$ \\
\hline Swift/BAT & Hard X-ray & $(8)$ & 246 & $\begin{array}{c}8 \\
0.03 \pm 0.01\end{array}$ & 152 & $\begin{array}{c}21 \\
0.09 \pm 0.02\end{array}$ & $\begin{array}{c}86 \\
0.36 \pm 0.03\end{array}$ \\
\hline $\begin{array}{l}\text { Swift/BAT } \\
\text { corrected }^{\mathrm{e}}\end{array}$ & & & 332 & $\begin{array}{c}8 \\
0.02 \pm 0.01\end{array}$ & 152 & $\begin{array}{c}21 \\
0.06 \pm 0.02\end{array}$ & $\begin{array}{c}172 \\
0.53 \pm 0.03\end{array}$ \\
\hline
\end{tabular}

a "Type L" is intended to refer to low excitation objects, but note that different authors define this class differently - by weak OIII emission, by LINER-like spectrum, or by weak MIR emission.

${ }^{\mathrm{b}}$ The number of Type 1 objects includes any Type 1R objects.

${ }^{c}$ Version- 1 of the statistics from the Palomar sample places all LINERs in the Type L bin.

dVersion-2 places LINER-1s with the Type 1 AGN, and LINER-2s in the Type L bin.

e The number of Type 2 objects has been approximately corrected using the prescription of Risaliti et al. 1999, that $50 \%$ of all Type 2 AGN are Compton thick.

References. - (1) Willott et al. (2000); (2) Ogle et al. (2006); (3) Lacy et al. (2007); (4) Rush et al. (1993); (5) De Grijp et al. (1992); (6) Maiolino and Rieke (1995); (7) Ho, Filippenko and Sargent (1997); (8) Tueller et al. (2009). 
must be careful with terminology. The value derived from the Maiolino \& Rieke (1995) sample, $\mathrm{f}_{2}=0.56 \pm 0.1$, is consistent with Type 2 and Type 1 AGN occuring in equal numbers; but the sum of Type 2 and Type 1R AGN is $76 \%$ of the sample, suggesting that "obscured" AGN outnumber "unobscured" AGN by 3 to 1. Fairly small-seeming differences can lead to quite different conclusions.

The numbers from HFS illustrate another problem vividly. The HFS sample includes 157 objects classified as "LINER", but many of these have broad lines, and so are classified "LINER-1". Of the "LINER-2"s, some may be obscured LINER$1 \mathrm{~s}$, and some may not. If we exclude all objects with a LINER classification completely (Palomar $\mathrm{V} 1$ in Table 1 ) then we get $\mathrm{f}_{2}=0.59$, closely similar to other samples; if we include the LINER-1s as Type 1 (Palomar V2), then we get $f_{2}=0.31$; this is obviously an underestimate, but we don't know how many of the LINER-2s to include. Perhaps the safest conclusion is to stay safely clear of the very lowest luminosity objects.

\subsection{SDSS AGN}

Several recent papers have considered the relative luminosity distributions of Type 1 and 2 AGN based on samples selected from the SDSS survey, but they have produced inconsistent results in the derived behaviour versus the luminosity in the [OIII] 5007 line. At low luminosities, L(OIII) $10^{33-34} \mathrm{~W}$, Simpson (2005) finds $f_{2} \sim 0.8$, but Hao et al. (2005) and Netzer (2009) find $f_{2} \sim 0.5$. At high luminosities, L(OIII) $\sim 10^{35-36} W$, Simpson (2005) finds $f_{2} \sim 0.4$, Hao et al. (2005) finds $f_{2} \sim 0.25$, Reyes et al. (2008) find $f_{2}>0.6$, and Netzer (2009) finds $f_{2} \sim 0.5$. (Note that the luminosity range in the figures of Hao et al. (2005) differs from the other authors by a factor of 100 , which we assume is an error. For Netzer (2009) we have estimated $f_{2}$ using the histograms shown in Fig 1 of that paper). Netzer (2009) stresses that there are difficult selection effects in using these data, especially at the low luminosity end; this is likely to be the origin of these discrepancies. A re-analysis of the SDSS data is underway to address these issues (Kewley et al. in preparation).

\subsection{Hard X-ray selected AGN}

X-ray selected samples are not a reliable way to measure $f_{2}$, because of the bias produced by $\mathrm{X}$-ray absorption. (The referee has asked us to stress that this should be considered the opinion of the authors.) However it is important to consider them, not least because of persistent findings that X-ray obscuration varies with luminosity (Lawrence \& Elvis 1982; Ueda et al. 2003; Hasinger 2008) - see section 2.6. For broad-band flux limited surveys in soft or medium energy $\mathrm{X}$ rays (e.g. $0.5-2 \mathrm{keV}$ or $2-10 \mathrm{keV}$ ), with sources showing a wide range in $N_{H}$ and redshift, it is hard to correct for the effects of X-ray absorption. (This is for example clearly explained in Dwelly \& Page (2006) : see their Fig. 15). Samples selected in hard X-rays ( $>10 \mathrm{keV}$; eg Sazonov et al. (2007); Aiello et al. (2008); Tueller et al. (2008)) are much better, because these should only be missing so called "Compton-thick" objects (i.e. those with $\mathrm{N}_{H}>2 \times 10^{24} \mathrm{~cm}^{-2}$ ). How many such objects appear in flux limited samples depends not just on the X-ray column density but also on what fraction of the central luminosity is scattered back into the line of sight. Judging by the variation in $\mathrm{L}_{X} / \mathrm{L}_{O I I I}$, the scattered fraction varies by an order of magnitude (Bassani et al. 1999), and there are examples of so called "buried" objects where the scattered fraction appears to be much less than 1\% (Bassani et al. 1999; Ueda et al. 2007; Sazonov et al. 2008). Risaliti et al. (1999) quantified the "missing objects" problem by examining X-ray spectra of a sample of optically selected AGN, finding that $50 \%$ of all such optically defined Type 2 AGN are Compton thick. Sazonov et al. (2007), from spectra of hard X-ray sources found with INTEGRAL, find a somewhat lower number of thick sources - 10-15\% of all non-blazar AGN, i.e. perhaps a quarter of Type 2 AGN. However, their starting sample was hard X-ray based, so we do not know how many objects with very low scattered fractions will have failed to make it at all into the INTEGRAL catalogue. The Risaliti et al. (1999) result is therefore probably the best indication we have of how to correct for missing Compton thick objects.

Table 1 shows the results from the updated SWIFT/BAT hard X-ray catalogue (Tueller et al. (2008); ref 8), who provide Type $1 \mathrm{R}$ as well as Type 1 and Type 2 numbers. To these num- 
bers we apply the Risaliti et al. correction factor for likely missing Compton-thick objects (an extra Compton-thick object for every Compton-thin Type 2 AGN), giving an estimate of $f_{2}=0.53$. This value is uncertain both because of the Comptonthick correction, and because of possible variation with luminosity - see section 2.6.

\subsection{Luminosity dependence of $\mathbf{f}_{2}$}

It is important to know whether $f_{2}$ varies with luminosity or not. As noted in the subsections above, a luminosity effect in radio samples originally claimed by Lawrence (1991) was shown to be caused by the appearance at low luminosities of weak-lined low-excitation objects, which likely are not obscured Type 1 AGN, and that the true Type 2 fraction does not change with luminosity (Willott et al. 2000). Likewise, when LINERs are discounted, the Rush et al 1993 IRAS MIR sample shows Type 1 and Type 2 luminosity functions which are closely matched throughout their luminosity range. Samples at very low luminosity (eg the Palomar sample of Ho et al. (1997)) and at very high luminosity (eg the MIR/radio sample of Ogle et al. (2006)) agree in showing Type 1 and Type 2 AGN in approximately equal numbers. The situation is summarised in the lower panel of Fig. 1, where results from various surveys are superimposed on a common bolometric luminosity scale, estimated using the mean quasar spectral energy distribution from Elvis et al. (1994). (This bolometric comparison is only approximate, but for comparisons over 5 decades will be adequate). From the radio, IR, and volume limited samples there is no evidence of variation in $f_{2}$ over 5 decades in luminosity.

The situation is strikingly different for Xray samples. There are long standing claims that the prevalence of X-ray absorbed objects and/or Type 2 AGN changes with X-ray luminosity (Lawrence \& Elvis 1982; Ueda et al. 2003; Hasinger 2008), but there have also been claims that this effect is in fact not present (Dwelly \& Page 2006; Eckart et al. 2006). In the upper panel of Fig. 1 we compare the results from the hard X-ray sample of Tueller et al. (2008) with the result produced by Hasinger (2008) from a compilation of medium energy X-ray surveys. After making the correction for missing Comptonthick objects described in the previous section, they agree very well, and disagree clearly with the behaviour shown by MIR and volume limited samples. Gilli et al. (2007) show that such a variation of obscured fraction with X-ray luminosity produces an excellent fit to the $\mathrm{X}$-ray background and X-ray number counts, strongly suggesting that this is a real effect, not simply a problem with current studies. In principle, the X-ray background can also constrain the number of Compton-thick objects, but the discussion in Gilli et al. (2007) shows that this constraint is a very weak one, depending sensitively on the assumed scattered fraction.

However, objects known to be Compton thick are not completely absent from the Tueller et al sample - for example NGC 1068 is present. Wang \& Jiang (2006) have argued that after removing known or suspected Compton-thick objects, the correlation of obscuration with luminosity is much less significant. It is striking that NGC 1068 is present with an apparent luminosity two orders of magnitude less than its likely true luminosity, usually assumed to be due to a small fraction of the nuclear light scattering back into the line of sight. We note that Winter et al. (2009) find that half the objects in the Swift/BAT sample require a complex spectral fit, for example partial covering. This raises the interesting possibility that many observed objects are partly covered with Compton thick material, and partly covered by material of intermediate thickness; such objects would be seen as apparently "Compton thin" but with a suppressed X-ray luminosity. This would produce an artifical correlation of obscured fraction with X-ray luminosity, and so could potentially produce the discrepancy observed in Fig. 1. Whether this produces a quantitatively correct explanation will be explored in a separate paper (Mayo et al. in preparation.)

Another possibility for explaining the discrepancy in Fig. 1 is an effect due to evolution masquerading as a luminosity effect. In X-ray samples, the question of the evolution of the observed obscured fraction has been controversial, but the conclusion seems to be a moderate observed change of the order $(1+z)^{0.4}$ (see discussion and analysis in Hasinger (2008)). In the radio, Willott et al. (2000) find no significant evolution in the ratio of narrow-line to broad-line objects, but a mild evolution of the kind possibly 
seen in X-rays is hard to rule out. What about the IR ? In the lower pane of Fig. 1, most of the high luminosity points are from the IRAS sample of de Grijp et al. (1992), with very few objects at $z>0.1$, and some from the Spitzer sample of Lacy et al. (2007), with a median redshift of $z=0.6$, suggesting at most a $20 \%$ effect due to evolution of the strength possibly seen in X-ray samples. This does not then seem to the explanation of the discrepancy seen in Fig. 1; however it would be highly desirable to undertake a fuller study of the luminosity and redshift effects in MIR selected samples.

Another possible method of constraining the covering factor, at least in Type 1 AGN, is by asking what fraction of the bolometric luminosity emerges in the IR. The SED compilations of Elvis et al. (1994) and Sanders et al. (1989) show that this is typically $30 \%$, but with a large source-to-source variation. More recent studies of Type 1 AGN with Spitzer show that the ratio of mid-IR to optical luminosities is larger for lower luminosity AGN, and this has been interpreted as implying that the torus covering factor decreases from $\sim 90 \%$ at $\mathrm{L}_{b o l} \sim 10^{43} \mathrm{erg} \mathrm{s}^{-1} \sim$ to $\sim 20 \%$ at $\mathrm{L}_{b o l} \sim 10^{47} \mathrm{erg} \mathrm{s}^{-1}$ (Maiolino et al. 2007; Treister et al. 2008; Hatziminaoglou et al. 2009). However the derivation of covering factors from $\mathrm{L}_{I R}$ is highly model dependent, and there are likely selection effects such as starburst components being more dominant at low powers, and Type 1R AGN having artificially low apparent optical luminosities. The latest such study (Mor et al. 2009) fits more detailed models to IR SEDs, including a clumpy torus and a starburst component. They find a mean torus covering factor of 0.27 , and at best marginal evidence for an anti-correlation with luminosity.

There are some interesting and important effects which need further study, but IR, radio, and volume limited samples must be the most reliable. It therefore seems likely that the true value of $f_{2}$ does not change with luminosity, with as yet poorly understood selection effects causing an apparent trend in other studies.

\subsection{Conclusions on Type 2 fraction}

Table 1 summarises the results on $f 2$ for different samples, and Fig. 1 shows the evidence on luminosity variation on $f_{2}$. As discussed in section

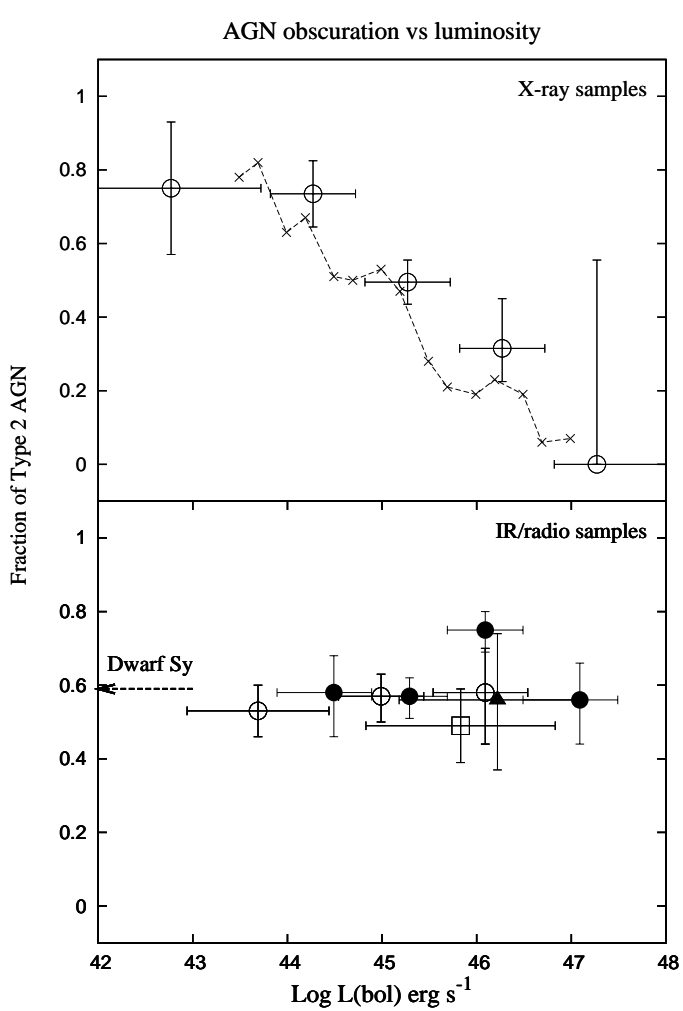

Fig. 1.-Behaviour of $f_{2}$ with estimated bolometric luminosity. Upper pane, open symbols - data from Tueller et al 2009; crosses - Hasinger 2008. Lower pane, filled circles - De Grijp et al 1992; open circles - Rush et al 1993; open squares - Lacy et al 2007; filled triangles - Ogle et al 2006; dashed line - Ho et al 199\%. Error bars are 68\% confidence except for the lower limit at high X-ray luminosities, which shows a $90 \%$ confidence interval.

2.6. the X-ray data show a luminosity dependence, but other selection methods do not, so it is reasonable to compare the non-X-ray samples. They are statistically consistent with a median value of $f_{2}=0.56$, a weighted mean value of $f_{2}=0.58 \pm 0.03$, and a dispersion of $\sim 0.05$. (Given the likely remaining systematic errors, the latter is a better indication of the uncertainty.) By contrast, the number of Type $\mathrm{L}$ objects varies widely between samples, depending on both the selection method and the luminosity range covered by a sample. The fraction of Type $1 \mathrm{R}$ objects is poorly known, but is approximately $0.15 \pm 0.05$. All in all, a reasonable statement on current evidence is that out of every 100 AGN, 30 are relatively unobscured, 15 
are lightly obscured, and 55 are heavily obscured.

There may be a continuous distribution of degrees of obscuration. Alternatively there may be physically distinct light and heavy obscuring agents. If these agents are independent and occur randomly, then the number of lightly obscured objects may be twice as many as indicated above, as heavily obscured AGN may have additional light obscuration which goes unnoticed. In this case out of every 100 AGN, 30 AGN are unobscured, 55 are seen through a heavy obscurer, and 33 are seen through a light obscurer, including 18 out of the 55 heavily obscured AGN. For example, Maiolino \& Rieke (1995) noted that moderate obscuration, unlike heavy obscuration, correlates with host galaxy inclination, suggesting that there are two separate obscurers, one nuclear and one host-related. (This effect can also be seen in more recent faint X-ray samples, e.g. Brand et al. (2007). In this picture, we would interpret the AGN Type statistics as indicating that the nuclear obscurer has a covering factor of $\sim 55 \%$, and the host-related obscurer a covering factor of $\sim 33 \%$.

In the remainder of this paper, we consider only the heavy obscurer, which is almost certainly nuclear, and has a covering factor of just over $50 \%$.

\section{Nuclear structures and gas flow}

In section 4 we look at predictions from a simple model of the obscuring region. In this section we examine evidence on the nature of the obscuring region in AGN, in order to constrain the ingredients of such a model.

\subsection{Location and size of obscurer}

What do we know about the material constituting the geometrically thick obscurer ? We can estimate its distance if we assume that the dust observed radiating is part of the same structure responsible for the extinction - a good assumption, but not a certain one. The IR SED typically peaks around 10-20 $\mu \mathrm{m}$ (Elvis et al. 1994) implying a characteristic radiating temperature of $\sim 200 K$. A minimum size is that of the blackbody producing this radiation, with $\mathrm{R}_{B B} \sim 3 \mathrm{~L}_{I R-44}^{1 / 2}$ $\mathrm{T}_{200}^{-2}$ pc where $\mathrm{L}_{I R-44}$ is the infrared luminosity in units of $10^{44} \mathrm{erg} \mathrm{s}^{-1}$. A maximum size is set by the distance at which an inefficient greybody dust grain is in equilibrium at $\mathrm{T}=200 \mathrm{~K}$ when exposed to the full unattenuated heating power : $\mathrm{R}_{e q}=37$ $\mathrm{L}_{44}^{1 / 2} \mathrm{~T}_{200}^{-2.8}$ pc (Barvainis 1987).

Realistically the size is likely to be in between these two, set by model dependent radiative transfer effects, i.e. self-shielding. Model fits indicate that optical depths around $\tau \sim 3$ are appropriate (Barvainis 1987), so that the dust is at $\sim 1.8$ $\mathrm{L}_{44}^{1 / 2} \mathrm{~T}_{200}^{-2.8}$ pc. However, reverberation measurements in Seyfert galaxies at $2 \mu \mathrm{m}$ show delays on timescales of 10-100 days implying far smaller sizes (Suganuma et al. 2006), which must therefore relate to much hotter dust. At an assumed sublimation temperature of $T \sim 1500 \mathrm{~K}, \mathrm{R}_{e q}=0.13$ $\mathrm{L}_{44}^{1 / 2}$ pc, equivalent to light travel time $\mathrm{t}=155 \mathrm{~L}_{44}^{1 / 2}$ days. This is just consistent with the observed delays, but inconsistent with the SED peak. AGN therefore contain dust at a wide range of temperatures, covering distances ranging from $0.1 \mathrm{pc}$ to $10 \mathrm{pc}$ (at a fiducial luminosity of $10^{44} \mathrm{erg} \mathrm{s}^{-1}$ ). However, hot dust far outshines cool dust per unit area, at all wavelengths; for the SED to peak at $10-20 \mu \mathrm{m}$, the bulk of the radiating area must be at $\sim 1$ pc. This dominance of the $\sim$ pc scale dust could be produced by geometric or self shielding effects, or both. If the luminosity is produced by accretion onto a black hole radiating at fraction $\epsilon$ of its Eddington luminosity, then we can express this characteristic size scale as

$$
R / R_{S} \sim 1.5 \times 10^{6} e^{-\tau / 3}(\epsilon / 0.1) L_{44}^{-1 / 2} T_{200}^{-2.8}
$$

where $\mathrm{R}_{S}$ is the Schwarzschild radius, $\epsilon$ is taken to be 0.1 , and the dust optical depth is taken to be $\tau=3$.

Current mid-infrared interferometric measurements, with $\sim 10$ mas resolution, can just resolve such scales in the very nearest AGN, and confirm parsec size scales directly in NGC 1068 and Circinus (Jaffe et al. 2004; Tristram et al. 2007; Raban et al. 2009). Cold gas is also detected via maser emission, and in a handful of cases has been beautifully resolved by VLBI measurements - in NGC 1068 Greenhill \& Gwinn 1997; Gallimore et al. 2004), in NGC 4258 (Greenhill et al. 1995; Mivoshi et al. 1995; Herrnstein et al. 2005; Humphreys et al. 2008), and in Circinus (Greenhill et al. 2003). These observations are consistent with thin Keplerian disks at variously convincing levels, from NGC4258 (convincing) to NGC1068 (possible). The discs are warped but not very 
strongly so; we need to be careful of the selection effects that make masers visible. Some discussions (e.g. that in Raban et al. (2009)) assume that the masers are showing the outer edge of the accretion disc, with the puffed-up "torus" lying just outside this, but those working on the maser observations themselves have suggested that the observed warped disc itself produces the obscuration (Greenhill et al. 2003; Herrnstein et al. 2005; Fruscione et al. 2005). NGC 1068 also shows a cold warped disk structure in $\mathrm{CO}$ emission (Schinnerer et al. 2000) but this is on considerably larger scales, 50-100 pc. Finally, an intriguing new observation of molecular $\mathrm{H}_{2}$ in NGC 1068 has been made by Sanchez et al. (2009) using Adaptive Optics and IR integral field spectroscopy, on a scale of $10 \mathrm{pc}$. This shows neither a disk-like structure, nor a "torus", but two linear streamers apparently pointing to and moving towards the nucleus.

\subsection{Orientation of obscurer}

What do we know about the orientation of the cold gas with respect to the accretion disk and black hole spin axis ? In NGC 1068, NGC 4258, and Circinus, the parsec scale maser and MIR material are oriented at least roughly perpendicularly to the radio axis (Greenhill et al. 2003; Herrnstein et al. 2005; Raban et al. 2009). On the other hand, the radio axis seems to be randomly oriented with respect to the host galaxy in Seyfert galaxies (Ulvestad \& Wilson 1984; Clarke et al. 1998). The situation with radio galaxies has been more confused. Schmitt et al. (2002) describe the debate on this issue, but conclude that the radio axis is randomly oriented with respect to the kpc scale dust disks often found in the elliptical host galaxies. For both Seyferts and radio galaxies however, the data are also consistent with being random over a large polar cap (Kinney et al. 2000; Schmitt et al. 2002). A recent large statistical study of radio sources coincident with SDSS galaxies (Battye \& Browne 2009) found clear evidence that the radio major axis is "biased towards" being aligned with the optical minor axis in early type galaxies, but this is seen as a $\sim 15 \%$ excess of aligned galaxies, consistent with the distribution being random to first order.

So it seems that the AGN itself has a well defined axis on parsec scales and below, but that this axis is, to first approximation, unconnected with the kpc scale structure it finds itself in.

\subsection{Gas flow in nuclear region}

What motion do we expect of gas reaching parsec scales? Central molecular discs in galaxies are turbulent and have scale heights of the order 100 pc (e.g. Scoville et al. (1993)), one to two orders of magnitude larger than the gravitational sphere of influence of the black hole. Velocity mapping of $\mathrm{H}_{2}$ emission lines in AGN on 30 pc scales using adaptive optics (Hicks et al. 2009) shows net rotation but with a velocity dispersion of a similar size - i.e. very thick discs. Both in our own Galactic Centre and in nearby AGN, this observed molecular material is almost certainly in a large number of small dense clumps, so that the covering factor to the nucleus is of the order $1 \%$ (see discussion in section 4 of Hicks et al. (2009)). This 30pc scale thick disc is therefore not the "torus"; the nuclear obscuration must typically happen closer in.

On 10 parsec scales, the movement of clumps is likely to be dominated by turbulence rather than rotation, so that motion will be close to isotropic. A tiny fraction of clumps (those on quasi-radial orbits) would be captured into the black hole sphere of influence. This seems to be just the picture shown by recent simulations, which find accretion rates (of captured material) that are highly variable on timescales of $10^{5} \mathrm{yr}$, with fluctuations of two orders of magnitude (Wada \& Norman 2002; Wada \& Tomisaka 2004; Escala 2007; Nayakshin \& Cuadra 2007). This seems to be borne out by observations of NGC 1068 (Sanchez et al. 2009), which show molecular streamers on quasi-radial orbits unconnected with the central disc orientation, on a scale of 5-20pc.

A good working hypothesis is therefore that AGN are fuelled by material arriving from 10$100 \mathrm{pc}$ scales, in discrete events coming from random directions. Each event may create a well defined accretion disc, but the ensemble of events over a long timescle has no net direction.

\subsection{Nuclear warped discs}

The combination of a well-defined central accretion axis and material arriving from random directions on parsec scales suggests that a re-alignment of material must occur in between, producing ex- 
treme warping.

Warping of accretion discs has been discussed a number of times in the AGN literature. Pringle (1996) showed that a radiation pressure instability can induce extreme warping in self-illuminated discs. Navakshin (2005) showed that in the transition region between the $\mathrm{kpc}$ scale disc and the sphere of influence of the black hole, the massive outer disk produces a torque on the inner accretion disc, inducing strong warping on a few orbital timescales.

In contrast to these processes for inducing a warp, viscosity will try to keep a disc aligned over time (Bardeen \& Petterson 1975; Pringle 1992), although there has been dispute over whether the black hole aligns the disc or vice versa (King et al. 2005; Volonteri et al. 2007). The warp radius, where the inner aligned planar disc meets the outer misaligned disk, could be the location of the AGN obscuring structure, although current models place this at $\sim 10^{4} \mathrm{R}_{S}$, rather less than the size scale of the AGN obscurer discussed in the previous section $\left(\sim 10^{6} \mathrm{R}_{S}\right.$, see eqn- 1$)$.

The true dynamics, and the warp induction or reduction mechanism, are beyond the scope of this paper. However, the hypothesis of random fuelling direction alone makes strong predictions which are generic and testable. We now look at these predictions.

\section{Tests of misaligned disc models}

In this section we look at generic tests of the hypothesis that incoming material is randomly misaligned, and somehow adjusts - first looking at the distribution of covering factors and predicted value of $f_{2}$, and then at possible misalignment effects. We need to consider both the tilt of the warp - how the misalignment between the spin axes of the central engine and each annulus varies with radius, $\theta(\mathrm{R})$; and the twist of the warp - how the azimuth of the line of nodes changes with radius, $\phi(\mathrm{R})$. Note that the twist refers to the shape of the structure at an instant in time, not to the precession - in principle each annulus could be precessing but locked together. We can distinguish two extreme assumptions : $2 \pi$ twist, and zero twist.

\subsection{Covering factor for fully twisted discs}

If the twist is $2 \pi$ or more, a complete equatorial wall is formed; a symmetrical structure that would look like the traditional "torus". If the initial misalignment is $\theta$, then the covering factor is $C=\sin \theta$. The probability of a given $\theta$ is $d P=(\sin \theta / 2) d \theta$. We then find the distribution of covering factors to be

$$
d N=\frac{1}{2} \frac{C}{\sqrt{1-C^{2}}} d C
$$

Objects with large misalignments are more likely to be observed as Type 2 AGN; the distribution of covering factors for only those objects which appear to be Type 2 is $\mathrm{dN}_{2}=\mathrm{C} \times \mathrm{dN}(\mathrm{C})$, and the distribution for Type $1 \mathrm{~s}$ is $\mathrm{dN}_{1}=(1-$ $\mathrm{C}) \times \mathrm{dN}(\mathrm{C})$. The observed Type 2 fraction is then $f_{2}=\int \mathrm{dN}_{2}(\mathrm{C})$.

Fig 2 shows the distribution of $\mathrm{C}$ for objects with $\theta<\pi / 2$. Note that all objects with $\theta>\pi / 2$ (i.e. the predicted $50 \%$ where the incoming flow is counter-rotating) are completely obscured from all directions. Including all the counter-rotating cases $\left(\mathrm{f}_{2}=0.89\right)$, this model is in strong disagreement with the observed value of $f_{2} \sim 0.55$. Possibly the completely obscured counter-rotating cases would not even be recognised as AGN; they would perhaps be seen as ultraluminous IR galaxies, as suggested by many authors. However even ignoring the counter-rotating cases, this model makes too many obscured objects $\left(f_{2} \sim 0.8\right)$.

We can therefore reject the hypothesis of twisted warped discs with random incoming directions.

\subsection{Covering factor for tilt only discs}

For tilted discs with no twist, the covered sky is determined by the outermost annulus; this, together with interior annuli, defines a lune on the sky, so that the covering factor is $\mathrm{C}=\theta / \pi$ and the distribution of covering factors is

$$
d N=\frac{1}{2} \sin \pi C d C
$$

Fig. 3 shows the covering factor distributions. The result is that $f_{2}=0.5$ exactly; a value remarkably close to the observed value, with minimal assumptions. Note that the covering factor 


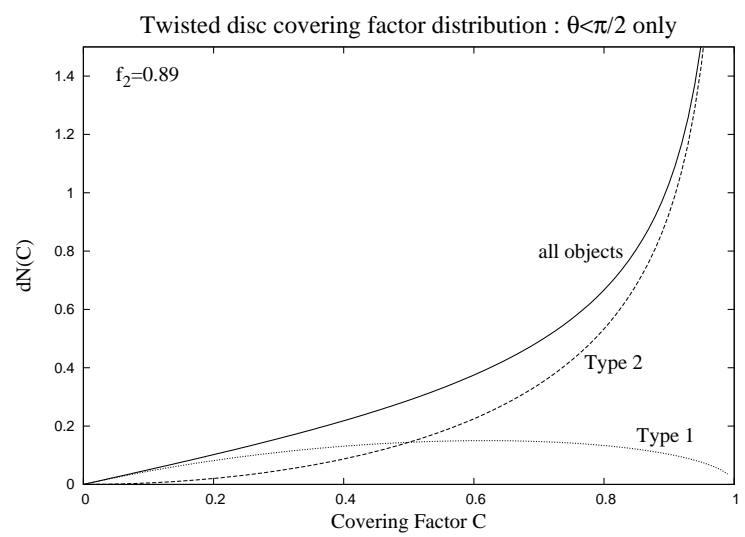

Fig. 2.- Covering factor distributions for a misaligned disc with random incoming rotation directions, where the line of nodes rotates at least one cycle during re-alignment.

distributions for objects seen as Type 1 and Type 2 are not the same. The typical covering factor for Type 1 AGN is $\sim 0.35$, close to the fraction of bolometric luminosity which emerges in the IR (Sanders et al. 1989; Elvis et al. 1994), and to the typical torus covering factor found in recent model fits (Mor et al 2009).

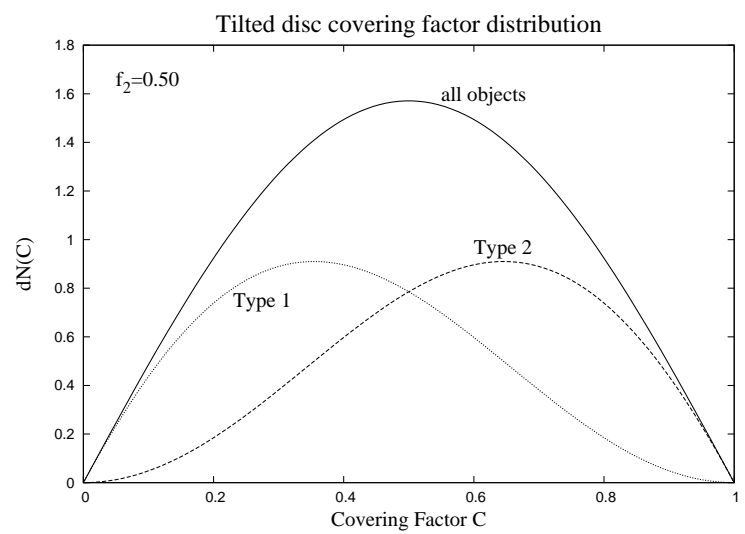

Fig. 3.- Covering factor distributions for a misaligned disc with random incoming rotation directions, where the line of nodes has no rotation during re-alignment.

\subsection{Intermediate cases}

Obviously more subtle parameterisations are possible; for example, a small amount of twist will increase the mean covering factor. Likewise fully

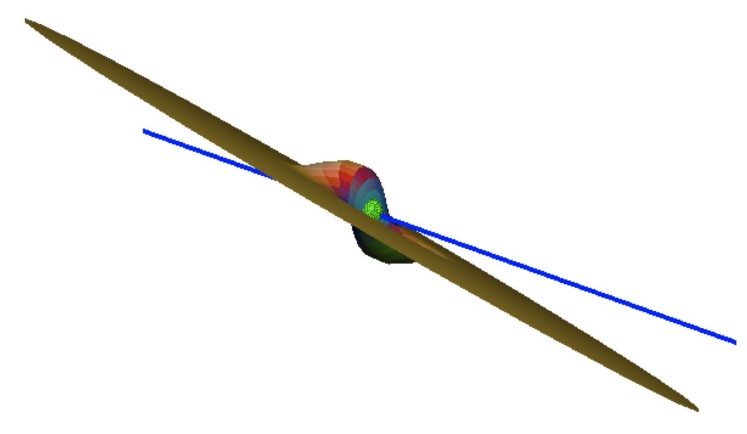

Fig. 4.- Illustration of what a tilted disc could look like seen almost edge-on, and close to the line of nodes. This example has an initial misalignment of $\theta=80^{\circ}$. The green ball represents the central source, and the blue line the radio jet.

twisted discs could be viable as a hypothesis if we give up the assumption of random misalignment. For example, if we assume that the probability of a given misalignment angle $\theta$ is smaller at large $\theta$ compared to a random distribution, e.g. tapered by a factor $\cos ^{2} \theta$, so that $d P=3 \sin \theta \cos ^{2} \theta d \theta$, then we find that the Type 2 fraction is $f_{2}=0.59$. However, a physical basis for such intermediate hypotheses would be needed before knowing what to test; we leave this to future work, and instead look at one more area of generic tests.

\subsection{Predicted misalignment effects}

We have seen above that randomly misaligned discs with no twist pass the covering factor test. However, such discs are not azimuthally symmetric. Although warped disk obscurers can produce shadow cones (Greenhill et al. 2003), and on average these will align with the nuclear axis, such a model predicts that distinct misalignments of nuclear structures will frequently be seen. An illustrative example is shown in Fig 4. In this section we test for such misalignments in a few exceptionally well observed individual cases, each one the brightest and nearest of its class - Type 1 Seyfert, Type 2 Seyfert, FRII radio galaxy. We find that interesting alignment anomalies are indeed present.

NGC 4151. Fig. 5 is a sketch indicating the structures seen in NGC 4151. As a Type 1 AGN, 
in the usual "torus" scheme, NGC 4151 should not show emission line cones at all, but it does (Hutchings et al. 1998). This situation is usually, though vaguely, explained as due to our observing at an angle grazing the torus. However, in the tilted disc picture, this combination is easy to achieve over a significant range of azimuths. The radio and emission line structures are roughly co-aligned, but the jet does not bi-sect the emission line structure (Mundell et al. 2003). Rather, it lies along one edge. There is a marginally resolved structure in molecular $\mathrm{H}_{2}$ (shown in blue in Fig. 5) which has been claimed as representing the "torus" in NGC 4151 (Fernandez et al. 1999) but this has a minor axis which differs from the radio axis by $\sim 80^{\circ}$.

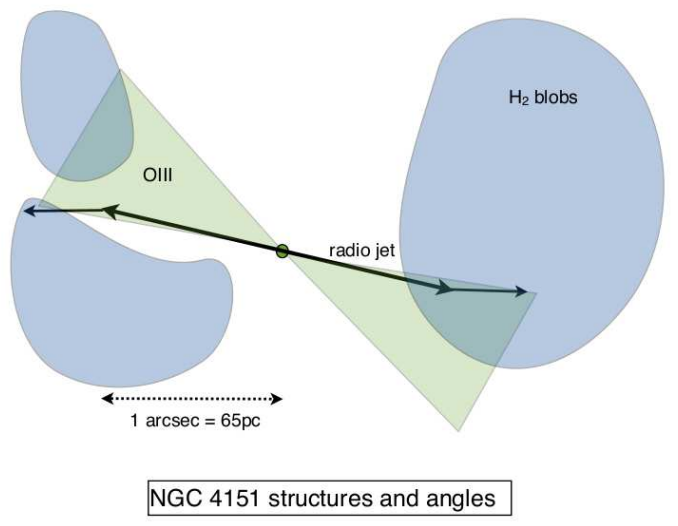

Fig. 5.- Sketch of structures seen in NGC 4151. The radio and emission line structures (black line and green region respectively) are taken from data and figures in Mundell et al. (2003); the $\mathrm{H}_{2}$ structures (blue regions) are taken from data and figures in Fernandez et al. (1999).

NGC 1068. This Type 2 object now has a rich variety of data. Fig 6 is a development of the illustration recently shown by Raban et al. (2009). (Note that this figure is not on a linear scale, and mixes structures on sub-pc and tens of pcs scales). The inner radio jet is orthogonal to the "hidden BLR" polarisation angle to within $5^{\circ}$, suggesting that both of these correctly indicate the (presumed) black hole spin axis. On sub-pc to $10 \mathrm{pc}$ scales, elongated structures are seen in all four of maser emission, mid-IR emission, radio continuum, and $\mathrm{H}_{2}$ emission, but the orientation changes systematically with radius. The minor axis is misaligned with the jet axis by $\sim 20^{\circ}$ on a scale of $\sim$ $0.1-0.4 \mathrm{pc}$ (inner maser disc), by $\sim 40^{\circ}$ on $\sim 0.4-$ $1 \mathrm{pc}$ scale (outer maser disc and mid-IR structure), and by $\sim 70^{\circ}$ on $1-10 \mathrm{pc}$ scales $\left(\mathrm{H}_{2}\right.$ streamers). The region as a whole seems to show incoming material approximately $\mathrm{N}-\mathrm{S}$ and a final major axis which is approximately E-W. Meanwhile the radio jet changes direction at "knot C", at a distance of $\sim 20 \mathrm{pc}$ from the nucleus (see Gallimore et al. (2004)). The outer jet is roughly aligned with the extended narrow emission-line region on $\sim 50-100$ pc scale, and both are misaligned with the inner radio jet by $\sim 20^{\circ}$

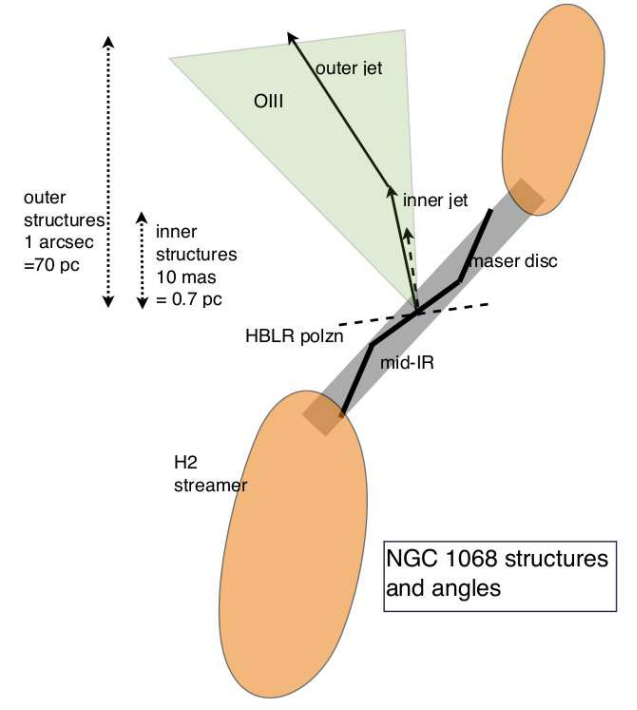

Fig. 6. - Sketch of structures seen in NGC 1068. (Note that this figure is not on a linear scale, and mixes structures on sub-pc and tens of pcs scales). The radio continuum and maser structures (black lines and dotted arrow) are taken from data and figures in Gallimore et al. (2004) and references therein; the mid-IR structures (grey region) are from Raban et al. (2009); the emission line structure (green region) are from Evans et al. (1991); the BLR polarization angle (dotted line) is from Antonucci \& Millen (1985); and the $\mathrm{H}_{2}$ structures (orange regions) are from Sanchez et al. (2009).

Cygnus A. In near-IR light, this object shows an impressive $\mathrm{X}$-shaped structure, suggestive of a hollow bi-cone, very well aligned with the radio axis, which is presumably made by scattered light (Tadhunter et al. 1999). However, IR polari- 
sation imaging shows that back reflection seems to occur only along one "wall" of that bi-cone (Tadhunter et al. 2000).

In conclusion, in well studied sources, there is clear evidence for misalignments, as expected for tilted disc obscurers. More detailed testing will require physical models. For example, scattered light "cones" will not be symmetric, but their surface brightness profiles will for example depend on how the density of scatterers varies with elevation.

\section{Discussion}

Here we briefly mention some further implications of misaligned discs, and especially of simple tilted discs, as AGN obscurers.

(i) In the misaligned disc picture, Type 1 and Type 2 AGN are still drawn from a single population, but they are not identical : Type 2 AGN are on average those objects with larger misalignments, and so larger covering factors. This potentially explains several interesting observations.

- The fraction of the bolometric luminosity of Type 1 AGN which emerges in the IR is smaller than the fraction of Type 2 AGN (Sanders et al. 1989; Elvis et al. 1994; Mor et al. 2009).

- Type 2 AGN on average have more prominent dust lanes (Malkan et al. 1998; Hunt \& Malkan 2004).

- Type 2 AGN have weaker narrow-line emission for a given radio power (Whittle 1985; Jackson \& Browne 1990; Lawrence 1991; Grimes et al. 2004); this could be because they have narrower opening angles, as they would if more tilted or twisted.

(ii) For both the twisted disc and tilted disc pictures, $50 \%$ of cases would result in any polar outflow (e.g. radio jet) colliding with the disc. The implications of this would depend on the mass of the disc compared to the momentum of the outflow. If the disc stops the outflow, then this predicts that not all Seyfert galaxies will have radio jets. If the outflow breaks through the disc, then the twisted disc model could be revived; incoming material would usually cover most of the sky, but the outflow produces a broken shell of obscuration. (This was suggested by Lawrence
(1991)). Jet-ISM collisions have of course often been discussed before, for example as an explanation for Compact Steep Spectrum (CSS) radio sources (see review by O'Dea (1998) and recent results by Guainazzi et al. (2006)). Jet-ISM interaction was proposed as the cause of the severe warp in 3C 449 (Tremblay et al. 2006), and has often been discussed in the context of the formation of the Narrow Line Region gas (Taylor et al. 1992; Bicknell et al. 1998; Dopita 2002).

(iii) We assumed for simplicity that incoming material has no effect on the spin of the central engine, but of course it will have, depending on how large each accretion event is. However, if incoming events arrive at random, then there will be no net spin-up. The fractional angular momentum change produced by each event (assumed to be of equal mass) will decrease with time as the black hole grows, so that the spin of the black hole does a random walk, gradually converging on a spin value which is well below maximal spin (Volonteri et al. 2007). It has been argued that the difference between radio loud and quiet $\mathrm{AGN}$ is in the spin of the black hole (Wilson \& Colbert 1995), and Sikora et al. (2007) specifically argue that while radio quiet $\mathrm{AGN}$ are indeed fed by many small accretion events from random directions, resulting in a low final spin, radio-loud AGN are fed by major mergers with a well determined angular momentum. If we can measure the distribution of tilt angles in AGN, then we may have an extra quantitative handle on these questions.

\section{Conclusions}

If one considers only reliable samples (i.e. those selected in the low frequency radio, in the mid-IR, or volume limited samples), removes low excitation objects, and includes lightly reddened objects with the Type 1 AGN, then the fraction of true, heavily obscured, Type 2 AGN is approximately $55 \pm 5 \%$. This value is independent of luminosity in IR, radio, and volume limited samples, but apparently changes with observed X-ray luminosity. The fraction of lightly reddened (but still recogniseably broad-line) AGN is 15-30\%, depending on whether this light obscuration occurs independently of the heavy obscuration. The heavy obscuration region is very likely the same as the parsecscale IR emitting region. 
Inflow from kpc scales to this parsec scale is likely to be in random directions, misaligned with the inner accretion flow, and thus should lead to severely warped structures. Random incoming discs with with complete twist produce too many obscured objects, but warps with no twist produce the correct fraction of Type 2 AGN. As, in this simple model, the mean tilt in Type $2 \mathrm{AGN}$ is larger than in Type 1 AGN, the relative strength of the IR and weakness of [OIII] are accounted for. This "tilted disc" picture also predicts significant misalignments of nuclear structures. Case studies of well resolved objects show that such misalignments do indeed occur.

\section{Acknowledgements}

This paper was written while AL was on sabbatical at KIPAC in Stanford, especially during a collaborative visit by ME. We would like to thank the staff of KIPAC for their hospitality and for the stimulating atmosphere. At various stages we have had very useful discussions with a number of colleagues that have helped to shape this paper : we would particularly like to thank Sterle Phinney, Andrew King, Reinhard Genzel, Ski Antonucci, Jean-Pierre Lasota, Marek Sikora, and Lukasz Stawarz. Responding to the robust criticisms of an anonymous referee also helped us to improve the paper considerably.

\section{REFERENCES}

Ajello, M., Rau, A., Greiner, J., Kanbach, G., Salvato, M., Strong, A. W., Barthelmy, S. D., Gehrels, N., Markwardt, C. B., \& Tueller, J. 2008, Astrophysical Journal, 673, 96

Antonucci, R. R. J., \& Miller, J. S. 1985, Astrophysical Journal, 297, 621

Ballantyne, D. R. 2008, Astrophysical Journal, 685,787

Bardeen, J. M., \& Petterson, J. A. 1975, Astrophysical Journal, 195, L65

Barvainis, R. 1987, Astrophysical Journal, 320, 537

Bassani, L., Dadina, M., Maiolino, R., Salvati, M., Risaliti, G., della Ceca, R., Matt, G., \& Zamorani, G. 1999, Astrophysical Journal Supplement Series, 121, 473
Battye, R. A., \& Browne, I. W. A. 2009, Monthly Notices of the Royal Astronomical Society (submitted : astroph-0902.1631)

Bicknell, G. V., Dopita, M. A., Tsvetanov, Z. I., \& Sutherland, R. S. 1998, Astrophysical Journal, 495,680

Brand, K., Dey, A., Desai, V., Soifer, B. T., Bian, C., Armus, L., Brown, M. J. I., Floc'h, E. L., Higdon, S. J., Houck, J. R., Jannuzi, B. T., \& Weedman, D. W. 2007, Astrophysical Journal, 663,204

Buchanan, C. L., Gallimore, J. F., O'Dea, C. P., Baum, S. A., Axon, D. J., Robinson, A., Elitzur, M., \& Elvis, M. 2006, Astronomical Journal, 132, 401

Carleton, N. P., Willner, S. P., Rudy, R. J., \& Tokunaga, A. T. 1984, Astrophysical Journal, 284,523

Chiaberge, M., Capetti, A., \& Celotti, A. 1999, Astronomy and Astrophysics, 349, 77

Clarke, C. J., Kinney, A. L., \& Pringle, J. E. 1998, Astrophysical Journal, 495, 189

de Grijp, M. H. K., Keel, W. C., Miley, G. K., Goudfrooij, P., \& Lub, J. 1992, Astronomy and Astrophysics Supplement Series, 96, 389

Dicken, D., Tadhunter, C., Axon, D., Morganti, R., Inskip, K. J., Holt, J., Delgado, R. G., \& Groves, B. 2009, Astrophysical Journal, 694, 268

Dopita, M. A. 2002, in Revista Mexicana de Astronoma y Astrofsica (Serie de Conferencias), Vol. 13, Emission Lines from Jet Flows, ed. W. Henney, W.Steffen, A. Raga, \& L.Binette, $177-182$

Dwelly, T., \& Page, M. J. 2006, Monthly Notices of the Royal Astronomical Society, 372, 1755

Eckart, M. E., Stern, D., Helfand, D. J., Harrison, F. A., Mao, P. H., \& Yost, S. A. 2006, Astrophysical Journal Supplement Series, 165, 19

Economou, F., Lawrence, A., Ward, M. J., \& Blanco, P. R. 1995, Monthly Notices of the Royal Astronomical Society, 272, L5 
Elitzur, M., \& Shlosman, I. 2006, Astrophysical Journal, 648, L101

Elvis, M., Marengo, M., \& Karovska, M. 2002, Astrophysical Journal, 567, L107

Elvis, M., Wilkes, B. J., McDowell, J. C., Green, R. F., Bechtold, J., Willner, S. P., Oey, M. S., Polomski, E., \& Cutri, R. 1994, Astrophysical Journal Supplement Series, 95, 1

Escala, A. 2007, Astrophysical Journal, 671, 1264

Evans, I. N., Ford, H. C., Kinney, A. L., Antonucci, R. R. J., Armus, L., \& Caganoff, S. 1991, Astrophysical Journal, 369, L27

Fabian, A. C., Barcons, X., Almaini, O., \& Iwasawa, K. 1998, Monthly Notices of the Royal Astronomical Society, 297, L11

Fernandez, B. R., Holloway, A. J., Meaburn, J., Pedlar, A., \& Mundell, C. G. 1999, Monthly Notices of the Royal Astronomical Society, 305, 319

Fruscione, A., Greenhill, L. J., Filippenko, A. V., Moran, J. M., Herrnstein, J. R., \& Galle, E. 2005, Astrophysical Journal, 624, 103

Gallimore, J. F., Baum, S. A., \& O'Dea, C. P. 2004, Astrophysical Journal, 613, 794

Gilli, R., Comastri, A., \& Hasinger, G. 2007, Astronomy and Astrophysics, 463, 79

Goodrich, R. W., \& Cohen, M. H. 1992, Astrophysical Journal, 391, 623

Greenhill, L. J., Booth, R. S., Ellingsen, S. P., Herrnstein, J. R., Jauncey, D. L., McCulloch, P. M., Moran, J. M., Norris, R. P., Reynolds, J. E., \& Tzioumis, A. K. 2003, Astrophysical Journal, 590, 162

Greenhill, L. J., \& Gwinn, C. R. 1997, Astrophysics and Space Science, 248, 261

Greenhill, L. J., Jiang, D. R., Moran, J. M., Reid, M. J., Lo, K. Y., \& Claussen, M. J. 1995, Astrophysical Journal, 440, 619

Grimes, J. A., Rawlings, S., \& Willott, C. J. 2004, Monthly Notices of the Royal Astronomical Society, 349, 503
Guainazzi, M., Siemiginowska, A., Stanghellini, C., Grandi, P., Piconcelli, E., \& Ugwoke, C. A. 2006, Astronomy and Astrophysics, 446, 87

Hao, L., Strauss, M. A., Fan, X., Tremonti, C. A., Schlegel, D. J., Heckman, T. M., Kauffmann, G., Blanton, M. R., Gunn, J. E., Hall, P. B., Ivezic, Z., Knapp, G. R., Krolik, J. H., Lupton, R. H., Richards, G. T., Schneider, D. P., Strateva, I. V., Zakamska, N. L., Brinkmann, J., \& Szokoly, G. P. 2005, Astronomical Journal, 129, 1795

Hardcastle, M. J., Evans, D. A., \& Croston, J. H. 2006, Monthly Notices of the Royal Astronomical Society, 370, 1893

-. 2009, Monthly Notices of the Royal Astronomical Society, submitted : astroph-0904.1323

Hasinger, G. 2008, Astronomy and Astrophysics, 490, 905

Hatziminaoglou, E., Fritz, J., \& Jarrett, T. 2009, Astronomy and Astrophysics, submitted : astro-ph0907.2389H

Herrnstein, J. R., Moran, J. M., Greenhill, L. J., \& Trotter, A. S. 2005, Astrophysical Journal, 629,719

Hicks, E. K. S., Davies, R. I., Malkan, M. A., Genzel, R., Tacconi, L. J., Sanchez, F. M., \& Sternberg, A. 2009, Astrophysical Journal (in press : astroph-0902.0978)

Hill, G. J., Goodrich, R. W., \& Depoy, D. L. 1996, Astrophysical Journal, 462, 163

Ho, L. C., Filippenko, A. V., \& Sargent, W. L. W. 1997, Astrophysical Journal, 487, 568

Hopkins, P. F., Hickox, R., Quataert, E., \& Hernquist, L. 2009, 0901.2936

Humphreys, E. M. L., Reid, M. J., Greenhill, L. J., Moran, J. M., \& Argon, A. L. 2008, Astrophysical Journal, 672, 800

Hunt, L. K., \& Malkan, M. A. 2004, Astrophysical Journal, 616, 707

Hutchings, J. B., Crenshaw, D. M., Kaiser, M. E., Kraemer, S. B., Weistrop, D., Baum, S., Bowers, C. W., Feinberg, L. D., Green, R. F., Gull, 
T. R., Hartig, G. F., Hill, G., \& Lindler, D. J. 1998, Astrophysical Journal, 492, L115

Jackson, N., \& Browne, I. W. A. 1990, Nature, 343,43

Jaffe, W., Meisenheimer, K., Rottgering, H. J. A., Leinert, C., Richichi, A., Chesneau, O., FraixBurnet, D., Glazenborg-Kluttig, A., Granato, G., Graser, U., Heijligers, B., Kuhler, R., Malbet, F., Miley, G. K., Paresce, F., Pel, J., Perrin, G., Przygodda, F., Schoeller, M., Sol, H., Waters, L. B. F. M., Weigelt, G., Woillez, J., \& de Zeeuw, P. T. 2004, Nature, 429, 47

King, A. R., Lubow, S. H., Ogilvie, G. I., \& Pringle, J. E. 2005, Monthly Notices of the Royal Astronomical Society, 363, 49

Kinney, A. L., Schmitt, H. R., Clarke, C. J., Pringle, J. E., Ulvestad, J. S., \& Antonucci, R. R. J. 2000, Astrophysical Journal, 537, 152

Krolik, J. H. 2007, Astrophysical Journal, 661, 52

Krolik, J. H., \& Begelman, M. C. 1986, Astrophysical Journal, 308, L55

—. 1988, Astrophysical Journal, 329, 702

Lacy, M., Petric, A. O., Sajina, A., Canalizo, G., Storrie-Lombardi, L. J., Armus, L., Fadda, D., \& Marleau, F. R. 2007, Astronomical Journal, 133,186

Laing, R. A., Jenkins, C. R., Wall, J. V., \& Unger, S. W. 1994, in ASP Conference Series, Vol. 54, The Physics of Active Galaxies, ed. G. Bicknell, M.A.Dopita, \& P.J.Quinn, 201

Lawrence, A. 1991, Monthly Notices of the Royal Astronomical Society, 252, 586

Lawrence, A. 2007, in Proceedings of IUA Symposium 238, Vol. 238, Black Holes from Stars to Galaxies - across the Range of Masses, ed. V.Karas \& G.Matt (Cambridge University Press), 117-122

Lawrence, A., \& Elvis, M. 1982, Astrophysical Journal, 256, 410

Maiolino, R., \& Rieke, G. H. 1995, Astrophysical Journal, 454, 95
Maiolino, R., Shemmer, O., Imanishi, M., Netzer, H., Oliva, E., Lutz, D., \& Sturm, E. 2007, Astronomy and Astrophysics, 468, 979

Malkan, M. A., Gorjian, V., \& Tam, R. 1998, Astrophysical Journal Supplement Series, 117, 25

Miyoshi, M., Moran, J., Herrnstein, J., Greenhill, L., Nakai, N., Diamond, P., \& Inoue, M. 1995, Nature, 373,127

Mor, R., Netzer, H., \& Elitzur, M. 2009, Astrophysical Journal, in press : astroph0907.1654M

Mundell, C. G., Wrobel, J. M., Pedlar, A., \& Gallimore, J. F. 2003, Astrophysical Journal, 583, 192

Nayakshin, S. 2005, Monthly Notices of the Royal Astronomical Society, 359, 545

Nayakshin, S., \& Cuadra, J. 2007, Astronomy and Astrophysics, 465, 119

O'Dea, C. P. 1998, Publications of the Astronomical Society of the Pacific, 110, 493

Ogle, P., Whysong, D., \& Antonucci, R. 2006, Astrophysical Journal, 647, 161

Osterbrock, D. E., \& Koski, A. T. 1976, Monthly Notices of the Royal Astronomical Society, 176, $61 \mathrm{P}$

Phinney, E. S. 1989, in NATO Advanced Science Institutes (ASI) Series C, Vol. 290, Theory of Accretion Disks, ed. F.Meyer (Dordrecht:Kluwer), 457

Pringle, J. E. 1992, Monthly Notices of the Royal Astronomical Society, 258, 811

-. 1996, Monthly Notices of the Royal Astronomical Society, 281, 357

Raban, D., Jaffe, W., Rottgering, H., Meisenheimer, K., \& Tristram, K. R. W. 2009, Monthly Notices of the Royal Astronomical Society (submitted : astroph-0901.1306)

Reyes, R., Zakamska, N. L., Strauss, M. A., Green, J., Krolik, J. H., Shen, Y., Richards, G. T., Anderson, S. F., \& Schneider, D. P. 2008, Astronomical Journal, 136, 2373 
Risaliti, G., Elvis, M., \& Nicastro, F. 2002, Astrophysical Journal, 571, 234

Risaliti, G., Maiolino, R., \& Salvati, M. 1999, Astrophysical Journal, 522, 157

Rush, B., Malkan, M. A., \& Spinoglio, L. 1993, Astrophysical Journal Supplement Series, 89, 1

Sanchez, F. M., Davies, R. I., Genzel, R., Tacconi, L. J., Eisenhauer, F., Hicks, E. K. S., Friedrich, S., \& Sternberg, A. 2009, Astrophysical Journal, 691, 749

Sanders, D. B., Phinney, E. S., Neugebauer, G., Soifer, B. T., \& Matthews, K. 1989, Astrophysical Journal, 347, 29

Sazonov, S., Revnivtsev, M., Burenin, R., Churazov, E., Sunyaev, R., Forman, W. R., \& Murray, S. S. 2008, Astronomy and Astrophysics, 487,509

Sazonov, S., Revnivtsev, M., Krivonos, R., Churazov, E., \& Sunyaev, R. 2007, Astronomy and Astrophysics, 462, 57

Schinnerer, E., Eckart, A., Tacconi, L. J., Genzel, R., \& Downes, D. 2000, Astrophysical Journal, 533,850

Schmitt, H. R., Pringle, J. E., Clarke, C. J., \& Kinney, A. L. 2002, Astrophysical Journal, 575, 150

Scoville, N. Z., Thakkar, D., Carlstrom, J. E., \& Sargent, A. I. 1993, Astrophysical Journal, 404, L59

Sikora, M., Stawarz, L., \& Lasota, J. 2007, Astrophysical Journal, 658, 815

Simpson, C. 2005, Monthly Notices of the Royal Astronomical Society, 360, 565

Suganuma, M., Yoshii, Y., Kobayashi, Y., Minezaki, T., Enya, K., Tomita, H., Aoki, T., Koshida, S., \& Peterson, B. A. 2006, Astrophysical Journal, 639, 46

Tadhunter, C. N., Packham, C., Axon, D. J., Jackson, N. J., Hough, J. H., Robinson, A., Young, S., \& Sparks, W. 1999, Astrophysical Journal, 512, L91
Tadhunter, C. N., Sparks, W., Axon, D. J., Bergeron, L., Jackson, N. J., Packham, C., Hough, J. H., Robinson, A., \& Young, S. 2000, Monthly Notices of the Royal Astronomical Society, 313, L52

Taylor, D., Dyson, J. E., \& Axon, D. J. 1992, Monthly Notices of the Royal Astronomical Society, 255, 351

Thompson, T. A., Quataert, E., \& Murray, N. 2005, Astrophysical Journal, 630, 167

Tozzi, P., Gilli, R., Mainieri, V., Norman, C., Risaliti, G., Rosati, P., Bergeron, J., Borgani, S., Giacconi, R., Hasinger, G., Nonino, M., Streblyanska, A., Szokoly, G., Wang, J. X., \& Zheng, W. 2006, Astronomy and Astrophysics, 451,457

Treister, E., Krolik, J. H., \& Dullemond, C. 2008, Astrophysical Journal, 679, 140

Tremblay, G. R., Quillen, A. C., Floyd, D. J. E., Noel-Storr, J., Baum, S. A., Axon, D., O'Dea, C. P., Chiaberge, M., Macchetto, F. D., Sparks, W. B., Miley, G. K., Capetti, A., Madrid, J. P., \& Perlman, E. 2006, Astrophysical Journal, 643, 101

Tristram, K. R. W., Meisenheimer, K., Jaffe, W., Schartmann, M., Rix, H., Leinert, C., Morel, S., Wittkowski, M., Rottgering, H., Perrin, G., Lopez, B., Raban, D., Cotton, W. D., Graser, U., Paresce, F., \& Henning, T. 2007, Astronomy and Astrophysics, 474, 837

Tueller, J., Mushotzky, R. F., Barthelmy, S., Cannizzo, J. K., Gehrels, N., Markwardt, C. B., Skinner, G. K., \& Winter, L. M. 2008, Astrophysical Journal, 681, 113

Ueda, Y., Akiyama, M., Ohta, K., \& Miyaji, T. 2003, Astrophysical Journal, 598, 886

Ueda, Y., Eguchi, S., Terashima, Y., Mushotzky, R., Tueller, J., Markwardt, C., Gehrels, N., Hashimoto, Y., \& Potter, S. 2007, Astrophysical Journal, 664, L79

Ulvestad, J. S., \& Wilson, A. S. 1984, Astrophysical Journal, 285, 439 
Urry, C. M., \& Padovani, P. 1995, Publications of the Astronomical Society of the Pacific, 107, 803

Volonteri, M., Sikora, M., \& Lasota, J. 2007, Astrophysical Journal, 667, 704

Wada, K., \& Norman, C. A. 2002, Astrophysical Journal, 566, L21

Wada, K., \& Tomisaka, K. 2004, in ASP Conference Series (ASPC), Vol. 320, The Neutral ISM in Starburst Galaxies., ed. S. Aalto, S. Huttemeister, \& A.Pedlar (Astronomical Society of the Pacific), 262

Wang, J. X., \& Jiang, P. 2006, Astrophysical Journal, 646, L103

Whittle, M. 1985, Monthly Notices of the Royal Astronomical Society, 213, 33

Whysong, D., \& Antonucci, R. 2004, Astrophysical Journal, 602, 116

Willott, C. J., Rawlings, S., Blundell, K. M., \& Lacy, M. 2000, Monthly Notices of the Royal Astronomical Society, 316, 449

Wilson, A. S., \& Colbert, E. J. M. 1995, Astrophysical Journal, 438, 62

Winter, L. M., Mushotzky, R. F., Reynolds, C. S., \& Tueller, J. 2009, Astrophysical Journal, 690, 1322

This 2-column preprint was prepared with the AAS LATEX macros v 5.2 . 